\title{
Experiencia de aprendizaje basado en problemas -Universidad De Colima
}

Ruíz Espinoza Francisco Higinio

fruiz@uabcs.mx

Roacho Contreras Silvia silroacho@ucol.mx

Ramírez Barrios Rafael alejandrocolion@hotmail.com

Salazar Dzul Sergio Iván sisalazar@hotmail.com

Soto Barrera Jenny sotojenny470@gmail.com

Tahuilan López Claudia clautahuilopez1978@hotmail.com

Vilchis Estrada Alejandra fbagens@hotmail.com Estudiantes de Doctorado en Educación Instituto de Estudios Superiores en Educación por Competencias Universidad de Colima

\section{RESUMEN}

La implementación de una nueva estrategia para impulsar el conocimiento de manera diferente con la finalidad del desarrollo de destrezas y competencias individuales en contextos formales con el beneficio por la interacción con otros, una de las estrategias utilizadas ha sido el aprendizaje basado en problemas (ABP), tanto para estudiantes de las áreas de la salud incurriendo después en otras áreas. En la Universidad de Colima esta estrategia se ha implementado desde aproximadamente 15 años. Los resultados de la Facultad de Trabajo Social de la Universidad de Colima respecto a la implementación del ABP, se concluyen experiencias positivas como negativas, entre las positivas se resalta el trabajo colaborativo del profesorado, permite tener un acercamiento mejor entre profesor-alumno, por lo que la evaluación debe ser un método más de enseñanza, y también una manera real y directa de posibilitar el aprendizaje. Por otra parte una de las negativas es la forma de evaluación debido a las rubricas donde se debe tomar en cuenta en el contexto laboral-docente, es el tipo de alumno que se tiene, los ritmos, estilos y motivos diferentes de aprendizaje. Además en el aula conviven estudiantes que llegan 
de diversas condiciones sociales, de hogares disfuncionales, algunos con paupérrimas condiciones de vida; otros de sociedades donde impera la delincuencia y todos los vicios sociales. El trabajo con los estudiantes requiere un manejo pedagógico y psicológico y mover estructuras mentales necesita constante preparación.

Palabras clave: Educación, aprendizaje centrado en el estudiante, innovación educativa, metodología de la enseñanza, competencias. 


\title{
Problem-based learning experience - Universidad De Colima
}

\begin{abstract}
The implementation of a new strategy to promote knowledge in a different way in order to develop individual skills and competencies in formal contexts with the benefit of interaction with others, one of the strategies used has been problem-based learning (PBL), so much for students of the areas of the health incurring later in other areas. At the University of Colima this strategy has been implemented for approximately 15 years. The results of the University of Colima regarding the implementation of the PBL, conclude positive and negative experiences, among the positive ones, the collaborative work of the teaching staff stands out, it allows to have a better approach between teacher-student, so the evaluation should be a more teaching method, and also a real and direct way of making learning possible. On the other hand, one of the negatives is the form of evaluation due to the rubrics where it must be taken into account in the work-teaching context, it is the type of student that one has, the different rhythms, styles and reasons for learning. In addition, the classroom is home to students who come from different social conditions, from dysfunctional homes, some with very poor living conditions; others from societies where crime and all social vices prevail. Working with students requires pedagogical and psychological management and moving mental structures requires constant preparation.
\end{abstract}

Keywords: Education, student-centered learning, educational innovation, teaching methodology, competencies.

Artículo recibido: 15 febrero 2021 Aceptado para publicación: 8 marzo 2021

Correspondencia: fruiz@uabcs.mx Conflictos de Interés: Ninguna que declarar 


\section{INTRODUCCIÓN}

Las deficiencias de la metodología de enseñanza tradicional y el nuevo enfoque que se deriva del Espacio Europeo de Educación Superior (EEES), en donde se incluye el desarrollo de competencias en las que hay que formar y evaluar, comporta la necesidad de introducir cambios en las estrategias instruccionales imperantes en las aulas universitarias (Prieto et al., 2006). Es bien conocido que las investigaciones en didácticas específicas, que cuestionan los modelos instruccionales habituales en la universidad, han tenido poco reflejo en la docencia universitaria (Mosquera y Furió, 2008).

Por lo que el desarrollo de destrezas y competencias individuales en contextos formales se ve beneficiado por la interacción con otros. No basta sólo con presentar determinadas actividades de aprendizaje, es necesario organizar el contexto en el cual el aprendizaje ocurre con el fin de optimizar el proceso y potenciar las destrezas de los alumnos (Arens, 2005).

En este sentido, en la búsqueda de un método didáctico generador de conocimientos significativos y de habilidades, se presenta el Método de Aprendizaje Basado en Problemas (ABP) como una alternativa efectiva para ser implementada en carreras de universitarias (Jofré et al., 2013).

El aprendizaje colaborativo es un enfoque clave para el desarrollo de actividades formativas. Para fomentar la colaboración entre los estudiantes, el aprendizaje basado en Proyectos (ABP) es un enfoque que fomenta la adquisición de competencias por parte de los estudiantes, ya que requiere que estos resuelvan actividades para el desarrollo de un producto concreto (Gómez et al., 2020). De esta manera Servín (2018) menciona que la implementación de una nueva estrategia para impulsar el conocimiento de manera diferente a estudiantes del área de la medicina incurriendo después en otras áreas, ha permitido a permitido como le menciona Servin. El mismo Servín establece que el aprendizaje basado en problemas (ABP) proporciona las bases de las habilidades para el descubrimiento, mediante un proceso heurístico, que ayuda a los estudiantes a internalizar el aprendizaje y los lleva a una mejor comprensión". Por tanto el ABP es una propuesta pedagógica para ayudar a la formación de los estudiantes de los diversos niveles académicos del siglo XXI, centrando el aprendizaje en las realidades prácticas y en una actitud constructiva de sus propios aprendizajes, creencias y aptitudes.

Por su parte George H. Watson, miembro del comité internacional del ABP de Estados Unidos, define el Aprendizaje Basado en Problemas como "un enfoque de aprendizaje que alienta a los estudiantes a aprender a aprender, trabajando de forma cooperativa mediante grupos, para buscar entre todas soluciones a problemas del mundo real" (Boletín informativo U de C, 2018). 
En este mismo sentido Rivera-Parada (2006) menciona que la la Red Panamericana para el Aprendizaje Basado en Problemas (PAN-ABP) es un método dinámico de enseñanza que viene extendiéndose en todo el mundo, tanto en las instituciones de educación superior como en las de educación escolar primaria y secundaria. Este modelo adapta "per se" la investigación como herramienta esencial; los estudiantes construyen sus propios conocimientos mediante la búsqueda de información para el estudio de problemas o necesidades y sus posibles soluciones creando un desafío para ellos. El ABP es una de las mejores formas de integrar la investigación y la docencia en los ámbitos sociales, de manera que promueve una formación de profesionales integrales para desenvolverse en la vida.

\section{Principales características del aprendizaje basado en problemas}

De la diversidad de experiencias expuestas por las diferentes universidades y autores de los diversos países que han trabajado con esta metodología del $\mathrm{ABP}$, han concluido como características las siguientes:

- Es un método de enseñanza activo centrado en el alumno.

- Es un enfoque curricular, es decir, una manera de elaborar y organizar el currículo por problemas en lugar de por disciplinas.

- Se considera que es condición del aprendizaje que el estudiante se responsabilice de él.

- Utiliza estrategias de motivación intrínseca para que el alumno se sienta estimulado a buscar conocimientos por sí solo.

- El profesor deja de tener un papel protagonista para centrarse en aportar el apoyo requerido.

- Es una metodología de aprendizaje que favorece el trabajo en equipo y las relaciones interpersonales.

- Parte de un problema que permite demostrar los conocimientos previos de los estudiantes y externar aquellos que desconocen para resolverlo.

- Se nombra un alumno que funja el rol de coordinador o dinamizador del grupo y a otro que desempeñe el papel de secretario.

- Existe un inicio, un desarrollo y un cierre del problema planteado.

- Se trabaja con pequeños grupos, los cuales varían de 7 a 12 estudiantes dependiendo de la universidad y la disciplina.

- Enfrenta a los estudiantes a dificultades reales que tienen que resolver aplicando los conocimientos y habilidades que ya poseen y otros nuevos que han de adquirir. 
- Al inicio de su formación del estudiante no debe esperarse que sea capaz de resolver problemas ya que su base de conocimiento no es suficiente para hacerlo, en la medida de su progreso se espera que estos sean resueltos.

- Los problemas que se le presentan al alumnado van dirigidos a adquirir conocimientos y no se espera que necesariamente estos sean resueltos.

- Permite demostrar efectivas habilidades de comunicación oral y escrita.

- Valora la importancia de recursos funcionales a disposición.

- Asigna un rol preponderante al contexto de los estudiantes y a la oportunidad de la experiencia concreta.

- Permite el desarrollo de competencias complejas, • Exige la aplicación de pasos lógicos de la investigación.

- Valora el carácter interactivo y el trabajo colaborativo, • Permite establecer relaciones de significado entre el interés personal, de estudios y de aplicación.

- Valora la importancia de un resultado, y

- Permite la reflexión sobre lo que se está aprendiendo mediante la solución del problema.

\section{Proceso del aprendizaje basado en problemas}

Existen diferentes formas de llevar a cabo el ABP, sin embargo tiene como eje principal trabajar con grupos pequeños asignado con un tutor, un problema a resolver, el estudiante es el protagonista principal de su propio aprendizaje y se registran los acontecimientos de las sesiones para dar continuidad al objetivo del problema y la solución a este. Uno de los que manejaron el proceso de esta estrategia fueron Torp y Sage (1988), quienes establecieron ocho pasos de importancia (Figura 1). 


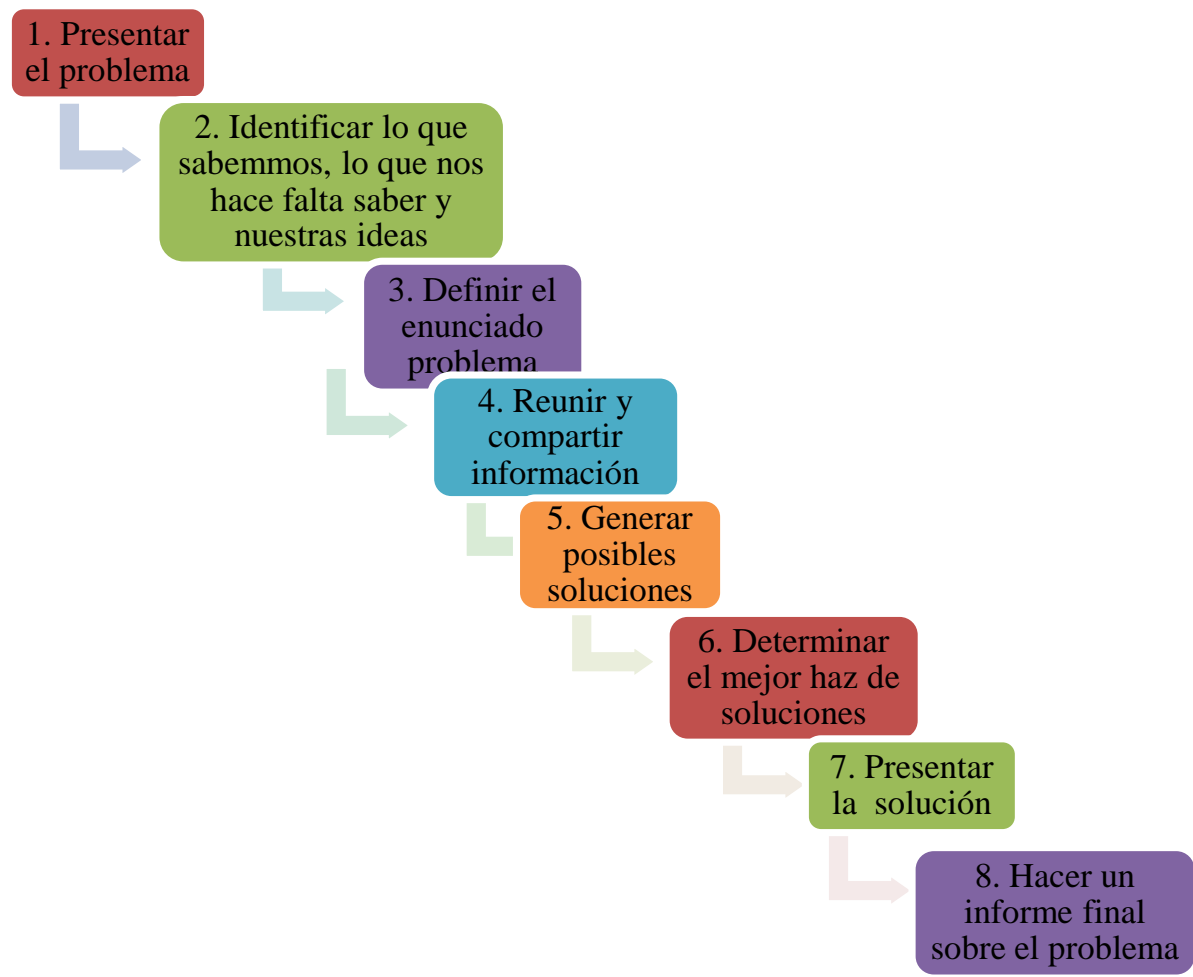

Figura 1. Esquema del proceso del la estrategia de aprendizaje basado en problemas

Fuente: Adaptado de Torp y Sage (1988).

Por otra parte Schmidt (1989) menciona que son siete pasos para el proceso o diseño del ABP (Figura 2)
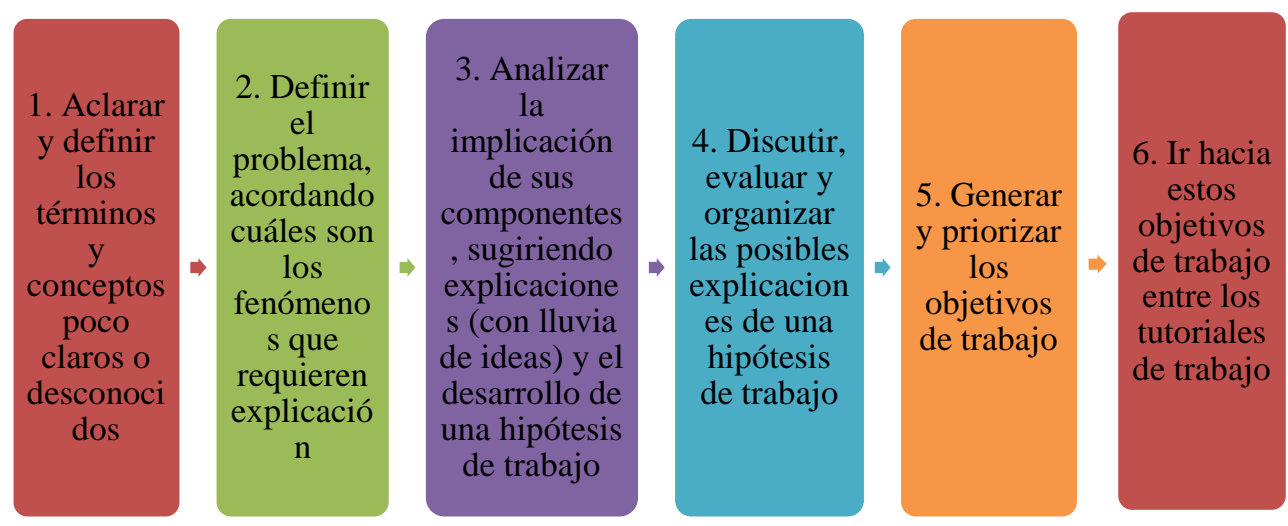

7. Llevar un informe en el siguiente tutorial con la síntesis sobre la explicación de todos los fenómenos y reaplicar esta síntesis al problema.ajo

Figura 2. Esquema del proceso del la estrategia de aprendizaje basado en problemas Fuente: Adaptado de Schmidt (1989). 
En tanto Servín (2018), menciona otros criterios como diseño para realizar el ABP (Figura 3), entre ellos están:

1. La planeación para el desarrollo de competencias y aprendizajes, partiendo de los aprendizajes esperados, es decir tener presente lo que lograrán los estudiantes y también como se valorará.

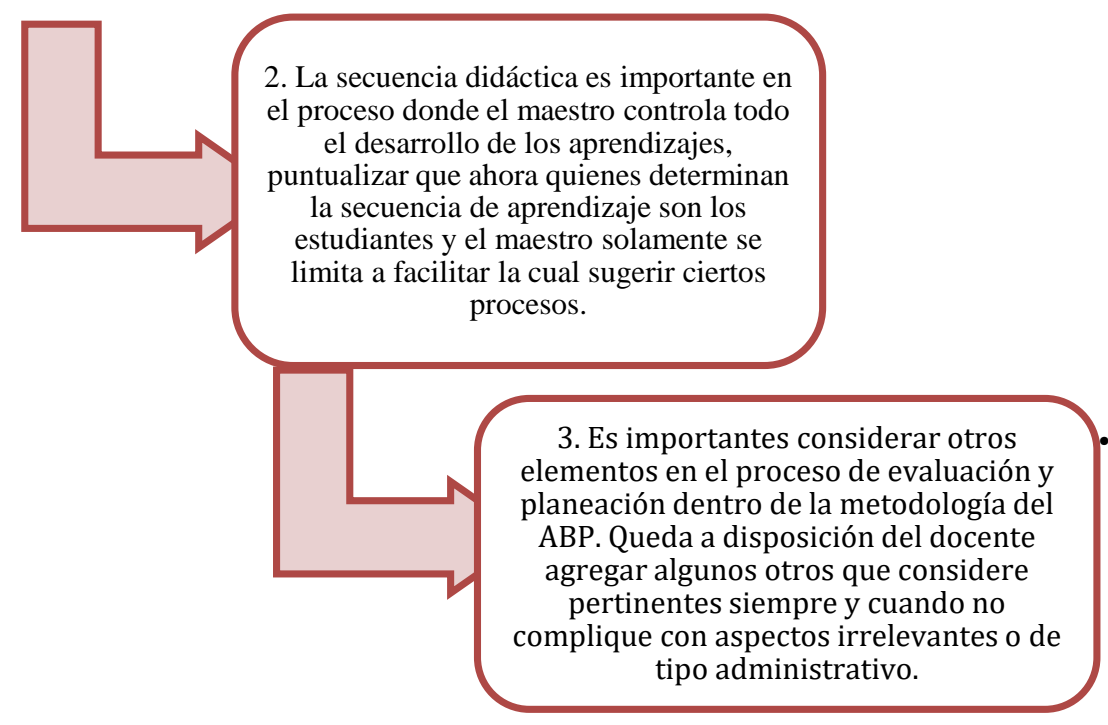

- Estos elementos son: a) el o los aprendizajes esperados; b) las temáticas o tópicos curriculares a considerar c) el problema planteado; d) preguntas que posibiliten la investigación y reflexión; e) sugerencia de actividades para los grupos colaborativos y para el facilitador; f) las rúbricas que valorarán los desempeños.

Figura 3. Esquema del proceso del la estrategia de aprendizaje basado en problemas Fuente: Servín (2018).

\section{Experiencias y resultados de su uso en diferentes niveles educativos}

Entre las experiencias que se encuentran sobre esta estrategia la Universidad Autónoma de Madrid menciona sus experiencias los cuales emprendieron esta metodología en la formación de los estudiantes de Magisterio de Educación Infantil de segundo curso. El grupo conformado por unos cien estudiantes se dividió en equipos de diez con un profesor fungiendo el rol de tutor. Previo a ello los profesores involucrados se les capacito sobre la metodología ABP, lo que les llevó a planificar y preparar los materiales necesarios para llevarla a la práctica. Entre los materiales diseñados fue enlistar las competencias generales que los alumnos deben desarrollar, precisando las habilidades de comunicación interpersonal, autonomía, búsqueda de conocimiento, capacidad crítica y trabajo cooperativo (Egido, 2006).

Por otra parte, el grupo de profesores consideran medular el problema que se le brinda a los estudiantes el cual debe poseer una estructura que les permita generar el punto de partida del proceso de un aprendizaje, es decir, de las competencias que debe alcanzar el estudiante, así 
mismo que tenga relación con los conocimientos previos del alumno y que posea elementos situacionales de la vida real o profesional.

Otra de las experiencias registradas corresponde a la facultad de Medicina de la Universidad Nacional de Colombia, en la asignatura de Medicina Interna II; los docentes habían notado en los estudiantes de pregrado deficiencias en los siguientes aspectos: fragmentación del conocimiento, ritualización del saber y el interés de memorizar conceptos solo para responder exámenes. Ante este panorama se surge la necesidad de generar nuevas prácticas académicas que permitiera al alumnado responder a las exigencias de la medicina en un contexto real, por ello consideran como una viabilidad el un nuevo método de aprendizaje como lo es el ABP, para resolver con facilidad los problemas que le ofrece su ejercicio profesional.

Barrows (1986) describe la experiencia de dividir el grupos no mayor de 7 alumnos y cada uno con un tutor, en la primera sesión se les presentó un problema médico con una sintomatología específica. El grupo discute el problema generando una hipótesis inicial basándose en su experiencia o conocimiento previo sobre el tema, así mismo deben identificar datos relevantes y los objetivos específicos relacionados con el problema. La sesión no puede terminar hasta cuando cada estudiante ha expresado su hipótesis inicial y asumiendo la responsabilidad de investigar un tópico específico que identificó.

Posterior a ello, los estudiantes empiezan su proceso de aprendizaje auto-direccionado, iniciando su búsqueda bibliográfica, consulta de libros, revistas electrónicas, bases de datos y opiniones de expertos. Cabe mencionar que fue necesario incorporar desde el inicio del proceso elementos de pensamiento crítico como un curso de Medicina Basada en Evidencia (MBE) para la cualificación de las fuentes (Sackett, 1996). El siguiente paso fue integrarse nuevamente los grupos para que los alumnos presentarán lo aprendido, valorar las fuentes consultadas y se cualifica lo útil y lo inadecuado, este proceso permitió revalorar el caso-problema con todos los elementos aportados por el resto del grupo generando nuevas hipótesis e identificando nuevos tópicos de interés y generando nuevas preguntas las cuales se resolverían con la nueva búsqueda de información en la siguiente sesión.

Otro aspecto a resaltar de esta experiencia fue la evaluación realizada en donde se responsabiliza el estudiante de auto evaluarse y emitir una evaluación de pares en tres áreas: auto-aprendizaje, capacidad de solucionar problemas y habilidades como miembro. En cuanto a los resultados de estas dos universidades descritas así como de otras más que han experimentado el ABP, se concluyen experiencias positivas como negativas, entre las positivas se resalta el trabajo colaborativo del profesorado, permite tener un acercamiento mejor entre profesor-alumno. 
La experiencia de trabajar con esta metodología abre las posibilidades de desarrollar una nueva metodología innovadora, permite un enfoque integrador e interdisciplinar que combina las perspectivas de diferentes asignaturas, constituye una oportunidad para trabajar en las competencias profesionales de los alumnos, plantea situaciones de aprendizaje acorde a la vida real, el alumno aprende a buscar y seleccionar información relevante para la solución del problema planteado, promueve la actitud cooperativa y dispuesta al intercambio que permite el desarrollo del sentimiento de pertenencia grupal, manejo eficiente de diferentes fuentes de información, comprensión de los fenómenos que son parte del entorno político, social, económico, ideológico, eentre otros, así como facilita la comprensión de los nuevos conocimientos, lo que resulta indispensable para lograr aprendizajes significativos.

Entre los aspectos negativos de la experiencia vivida por el grupo de profesores de la Universidad Autónoma de Madrid se resalta un alto costo de pago a personal dado que se trabaja con grupos pequeños, requiere de varios espacios (aulas) para desarrollar la estrategia dentro del plantel, así mismo de bibliografía para la consulta de los temas que necesitan revisar al mismo tiempo los diversos grupos conformados, requiere tiempo y dedicación sobre todo en la primera etapa del proceso tanto de alumnos como de profesores, dificultad en profesores y estudiantes para trabajar en equipo, resistencia al cambio por parte de profesores en dejar su rol del conocer y experto a ser solo guía o facilitador de conocimiento, algunos profesores tienden a orientar de manera indirecta desvirtuando la metodología del ABP.

\section{Diseñando propuestas del aprendizaje basado en problemas}

El ABP es una estrategia educativa que permite desarrollar en el alumno el razonamiento y el juicio crítico, cabe señalar que no es simplemente un método para facilitar el aprendizaje, sino representa una interpretación particular del proceso enseñanza-aprendizaje diferente a la implícita en la didáctica tradicional, por ello se han adoptado diversas definiciones de lo que es ABP y no todos se han ceñido a lo propuesto por Barrows (1986). La literatura médica está llena de publicaciones sobre los resultados al aplicar el ABP en uno u otro sentido, ello implica que tener como tal una sola propuesta de diseño del ABP es compleja por los fines que persigue el PE de cada carrera (medicina, psicología, trabajo social, etc.).

\section{Diseño de implementación Aprendizaje Basado en Problemas}

\section{Universidad de Colima (UC): Facultad de Trabajo Social}

En la Universidad de Colima el diseño de ABP se ha implementado desde aproximadamente 15 años en las Facultades del áreas de salud como medicina, psicología, enfermería y de las ciencias sociales en la facultada de trabajo social. 
La elección del modelo ABP adaptada por la Universidad de Colima para la práctica docente también viene precedida y suscitada por los resultados obtenidos en su utilización en distintos ámbitos. Sin es bien sabido que son bien conocidas las ventajas del modelo ABP en relación con la mejor consecusión y duración de una serie de competencias por los estudiantes formados mediante este modelo y en diferentes aplicaciones, tanto así en el desarrollo de habilidades del pensamiento crítico y al mayor grado de autoeficacia; por que las competencias autopercibidas son mejor comprendidas; asi mismo tambíen las competencias cognitivas y sociales; por otra parte la orientación intrínseca a los objetivos establecidos, a la valoración de las tareas, así como a las estrategias de elaboración, de la misma manera a la auto-regulación metacognitiva, y la regulación del esfuerzo y aprendizaje de iguales; por lo que las consecuencias proporcionadas a largo plazo en la adquisición de dichas competencias; y a su vez en el razonamiento clínico tratándose de medicina o enfermería (Şendağa y Odabaşi, 2009; Rajab, 2007; Cohen-Schotanus et al., 2008; Koh et al., 2008; Sungur y Tekkaya, 2010; Schmidt, Vermeulen y Van der Molen, 2006 y Scaffa y Wooster, 2004).

En la Figura 4 se esquematiza el proceso metodológico establecido y adaptado por la Universidad de Colima desde aproximadamente 15 años. 
-Se trabaja en grupos pequeños con un tutor responsable de guiar el proceso de aprendizaje del alumnado.

Paso 2

- Se designa en cada sesión un moderador y secretario

Paso 3

- Definición del Problema

Paso 4

- Lluvia de ideas

Paso 5

- Discusión y categorización de ideas

Paso 6

- Definición de los objetivos de aprendizaje

- Búsqueda de la información al recurrir a literatura seleccionada de fuentes confiables y entrevista a expertos acorde a la disciplina, esto permite plantearse nuevas hipótesis o se reafirman las ya expuestas, en el caso de las ciencias sociales se va construyendo un nuevo conocimiento en relación al problema, ambos casos permite criticar las teorías revisadas en función del contexto médico o social que se está discutiendo, así como estimula la discusión, el diálogo, la reflexión y la participación en la toma de decisiones.

-Evaluación continua por parte del propio estudiante (autoevaluación), de sus pares (evaluación de Paso 8 pares) y del tutor docente las cuales se realizan por medio de rúbricas.

- Reporte de resultados.

Figura 4. Esquema del proceso de la estrategia de aprendizaje basado en problemas en la Facultad de Trabajo Social Universidad de Colima

Fuente: Elaboración propia (2021).

Existen diferentes formas de llevar a cabo el ABP, sin embargo en la UC tiene como eje principal trabajar con grupos pequeños asignado con un tutor, un problema a resolver, el estudiante es el protagonista principal de su propio aprendizaje y se registran los acontecimientos de las sesiones para dar continuidad al objetivo del problema y la solución a este.

\section{Métodos de evaluación adaptado por la UC}

La evaluación educativa es un proceso continuo y personalizado dentro del sistema de enseñanza-aprendizaje cuyo objetivo es conocer la evolución de cada estudiante para, si es necesario, adoptar medidas de refuerzo o de compensación para garantizar que se alcanzan los objetivos educativos definidos para su nivel.

Según Mora (2004) la evaluación se puede entender de diversas maneras, dependiendo de las necesidades, propósitos u objetivos de la institución educativa, tales como: el control y la medición, el enjuiciamiento de la validez del objetivo, la rendición de cuentas, por citar algunos 
propósitos. Desde esta perspectiva se puede determinar en qué situaciones educativas es pertinente realizar una valoración, una medición o la combinación de ambas concepciones.

Las diferentes formas de evaluación tienen muchas aristas que pueden causar polémica, aunque se sabe que las rúbricas pueden causar diferencias por como se llevan a cabo, pero en la Universidad de Colima se decidió utilizar este tipo de evaluación, las rúbricas se utilizan como una herramienta para evaluar el aprendizaje del alumnado haciendo que los propios estudiantes también conozcan sus errores mediante la autoevaluación. La rúbrica en sí es un documento que describe distintos niveles de calidad de una tarea o proyecto, dando un feedback informativo al alumnado sobre el desarrollo de su trabajo durante el proceso y una evaluación detallada sobre sus trabajos finales.

A continuación, se presentan los formatos establecidos para evaluar las rúbricas.

Cuadro 1. Rúbrica: organizador gráfico

\begin{tabular}{|c|c|c|}
\hline Aspectos & Evaluación & Observaciones \\
\hline \multicolumn{3}{|l|}{ Identifica las ideas y conceptos relevantes. } \\
\hline \multicolumn{3}{|l|}{$\begin{array}{l}\text { Organiza la información de manera que permite una lectura clara } \\
\text { de la información. }\end{array}$} \\
\hline \multicolumn{3}{|l|}{$\begin{array}{l}\text { Si se trata de cuadro comparativo, identifica los elementos que } \\
\text { establecen la comparación. }\end{array}$} \\
\hline \multicolumn{3}{|l|}{ Si es mapa conceptual, deben identificarse los conectores. } \\
\hline \multicolumn{3}{|l|}{$\begin{array}{l}\text { Proposiciones escritas correctamente, atendiendo a las reglas } \\
\text { gramaticales }\end{array}$} \\
\hline \multicolumn{3}{|l|}{$\begin{array}{l}\text { Proposiciones escritas correctamente, atendiendo a las reglas } \\
\text { ortográficas }\end{array}$} \\
\hline \multicolumn{3}{|l|}{$\begin{array}{l}\text { Elabora correctamente la bibliografía de acuerdo con el código } \\
\text { APA. }\end{array}$} \\
\hline \multicolumn{3}{|l|}{ Subtotal } \\
\hline Calificación final & & \\
\hline
\end{tabular}

Escala: Excelente (1) Muy bien (0.8) Bien (0.6) Regular (0.4) Deficiente (0.2) No Aplica (NA):

Fuente: Facultad de Trabajo Social de la Universidad de Colima (2021) 
Cuadro 2. Rúbrica de evaluación para primer problema, listado de verificación de contenidos del portafolio

\begin{tabular}{l|l|l|}
\hline \multicolumn{1}{|c|}{ Estructura } & I & O \\
\hline 1.- Hoja frontal: que incluye datos de identificación de la misma: Universidad de & & \\
Colima, Facultad de Trabajo Social, nombre de la materia, nombre del trabajo, & & \\
nombre del estudiante, semestre, grupo, nombre del profesor, lugar y fecha (este & & \\
último alineado a la derecha). & & \\
2.- Índice: donde se indique el número de página de cada apartado. & & \\
3.- Problema: Incluir el problema motivo del portafolio. & & \\
4.- Objetivos de aprendizaje: se organizan en el orden en el que se jerarquizaron & & \\
por el grupo. & & \\
5.- Productos: & & \\
5.1 Organizador gráfico de cada objetivo & & \\
5.2 Reporte: descripción y análisis de la información consultada en donde el & & \\
estudiante integra todos los objetivos revisados, consultando diversas fuentes y & & \\
respetando el código APA, además de reflexiones personales en función del & & \\
problema que se está revisando (mínimo 2, máximo 4 cuartillas por unidad de & & \\
aprendizaje). & & \\
6.- Bibliografía (modelo APA y en orden alfabético) & & \\
7.- Anexos: (fichas de trabajo, fotografías, audio, material didáctico utilizado, & & \\
entre otros) & & \\
\hline
\end{tabular}

Fuente: Facultad de Trabajo Social de la Universidad de Colima (2021)

\section{Cuadro 3. Rúbrica de evaluación del Portafolio}

\begin{tabular}{|c|c|c|}
\hline CONTENIDOS & ESCALA & OBSERVACIONES \\
\hline \multicolumn{3}{|l|}{ 5. PRODUCTOS } \\
\hline \multicolumn{3}{|l|}{ 5.1 ORGANIZADORES GRÁFICOS } \\
\hline \multicolumn{3}{|l|}{$\begin{array}{l}\text { 5.1.1 Incluye el total de organizadores gráficos de los } \\
\text { objetivos revisados en las sesiones }\end{array}$} \\
\hline \multicolumn{3}{|l|}{$\begin{array}{l}\text { 5.1.2 Cada objetivo se refleja de manera clara en el } \\
\text { organizador gráfico }\end{array}$} \\
\hline \multicolumn{3}{|l|}{5.2 REPORTE } \\
\hline \multicolumn{3}{|l|}{$\begin{array}{l}\text { 5.2.1 La descripción incluye una diversidad de fuentes } \\
\text { consultadas: páginas web (3); libros y/o artículos } \\
\text { científicos de revista (3), experto (1). }\end{array}$} \\
\hline \multicolumn{3}{|l|}{ 5.2.2 Usa fuentes confiables } \\
\hline \multicolumn{3}{|l|}{$\begin{array}{l}\text { 5.2.3 Usa fuentes actualizadas (sólo en los datos históricos } \\
\text { de la familia se permitirán fuentes no actuales) }\end{array}$} \\
\hline \multicolumn{3}{|l|}{$\begin{array}{l}\text { 5.2.4 Identifica las ideas y conceptos relevantes de los } \\
\text { temas }\end{array}$} \\
\hline \multicolumn{3}{|l|}{$\begin{array}{l}\text { 5.2.5 Retoma los conceptos e ideas relevantes en la } \\
\text { comprensión de los temas }\end{array}$} \\
\hline $\begin{array}{l}\text { 5.2.6 Organiza e integra de manera lógica la información } \\
\text { presentada }\end{array}$ & & \\
\hline
\end{tabular}


5.2.7 Manifiesta su postura y opinión respecto a los distintos temas, incorporando el conocimiento adquirido.

\section{6.- ORTOGRAFÍA Y REDACCIÓN}

6.1 Proposiciones escritas correctamente, atendiendo a las reglas gramaticales

6.2 Proposiciones escritas correctamente, atendiendo a las reglas ortográficas

\section{7.- REFERENCIAS BIBLIOGRÁFICAS}

7.1 Realiza las citas bibliográficas atendiendo al código APA

7.2 Elabora correctamente la bibliografía de acuerdo con el código APA.

\section{Subtotal}

\section{Total}

Escala de evaluación: Excelente (2.5); Muy Bien (2); Bien (1.5); Regular (1)Deficiente (0.5); No

Aplica: Fuente: Facultad de Trabajo Social de la Universidad de Colima (2021)

Cuadro 4. Rúbrica para la evaluación de la participación del estudiante por parte del profesor

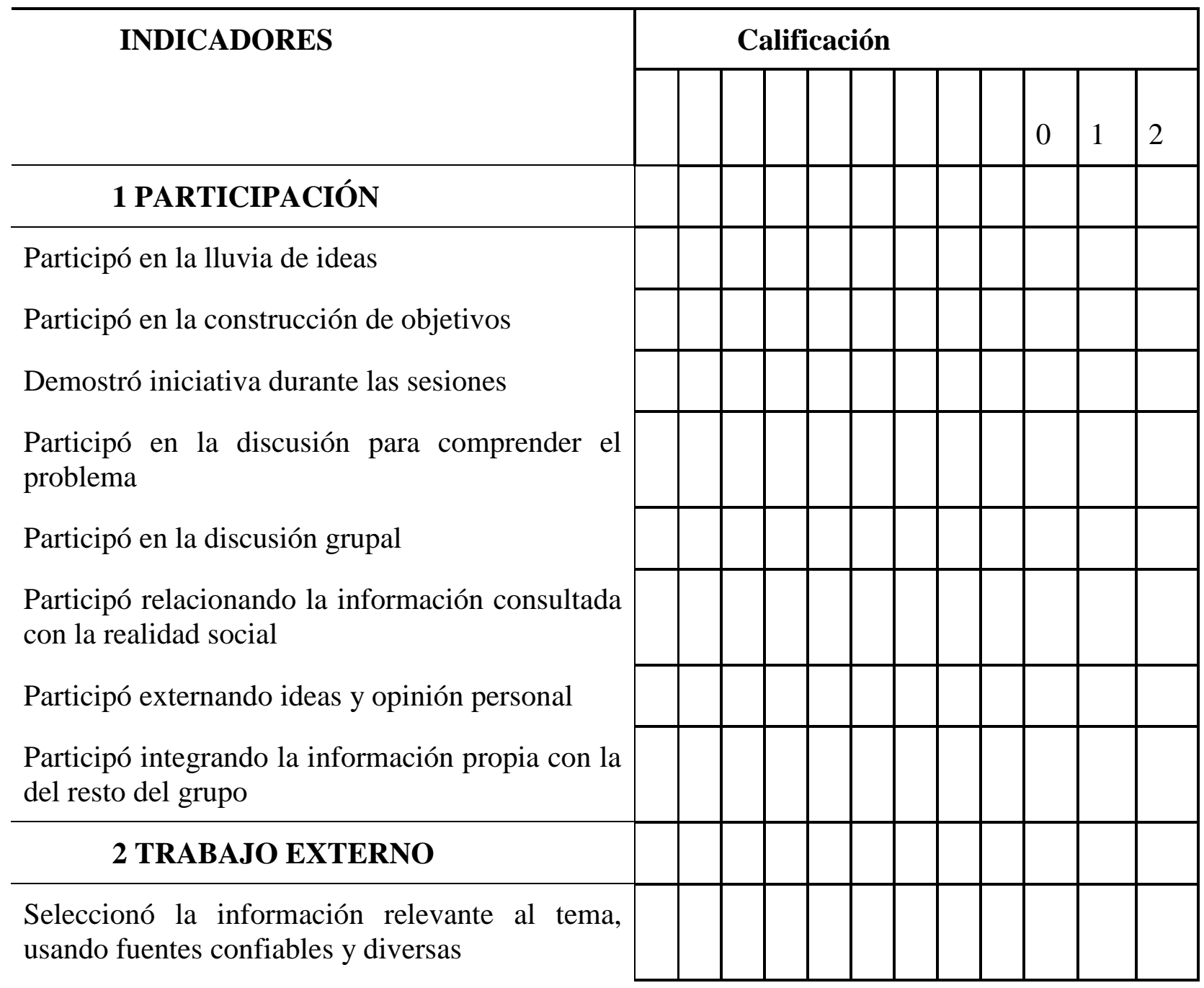


Elaboró las fichas de trabajo necesarias para cumplir con los objetivos

Realizó el organizador gráfico correspondiente con el objetivo de aprendizaje

\section{ACTITUDES}

Expresó ideas de manera clara, organizada y coherente

Fue responsable en los acuerdos establecidos por el grupo

Demostró compromiso y respeto con el trabajo del grupo

Utilizó un lenguaje profesional

Interactuó de manera respetuosa y profesional con el grupo

\section{Subtotal}

\section{Calificación final}

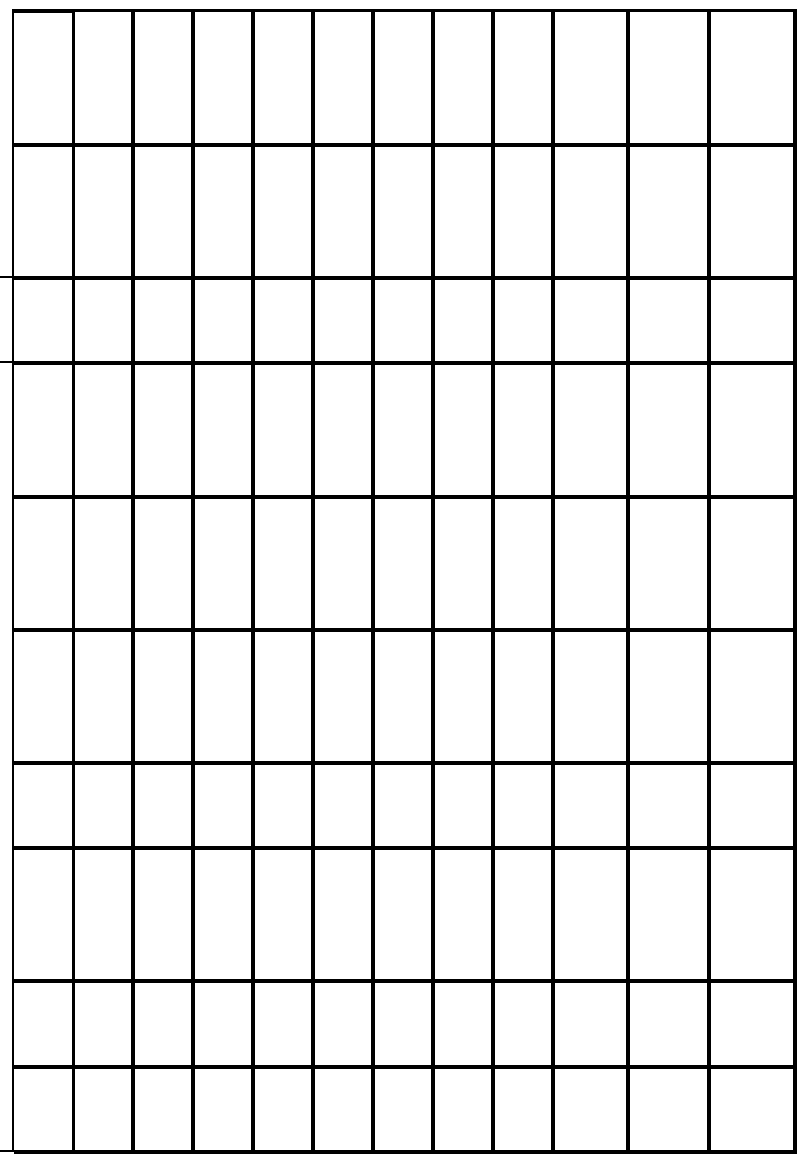

\section{RELACIÓN DE ESTUDIANTES}

\begin{tabular}{lll}
\hline 1.- & 2.- & 3.- \\
4.- & $5 .-$ & 6.- \\
7.- & 8.- & 9.- \\
10.- & $11 .-$ & $12 .-$ \\
\hline
\end{tabular}

Escala: Excelente (1.5) Muy bien (1.2) Bien (0.9) Regular (0.6) Deficiente (0.3) No Aplica (NA):

Fuente: Facultad de Trabajo Social de la Universidad de Colima (2021)

Cuadro 5. Rúbrica para la coevaluación

INDICADORES

\section{PARTICIPACIÓN}

Participó en la lluvia de ideas

Participó en la construcción de objetivos

Demostró iniciativa durante las sesiones

\begin{tabular}{|l|l|l|l|l|l|l|l|l|l|l|l|}
\hline \multicolumn{1}{|c|}{ Calificación } \\
\hline & & & & & & & & & & & \\
\hline & & & & & & & & & & & \\
\hline & & & & & & & & & & & \\
\hline & & & & & & & & & & & \\
\hline & & & & & & & & & & & \\
\hline
\end{tabular}


Participó en la discusión para comprender el problema

Participó en la discusión grupal

Participó relacionando la información consultada con la realidad social

Participó externando ideas y opinión personal

Participó integrando la información propia con la del resto del grupo

\section{TRABAJO EXTERNO}

Seleccionó la información relevante al tema, usando fuentes confiables y diversas

Elaboró las fichas de trabajo necesarias para cumplir con los objetivos

Realizó el organizador gráfico correspondiente con el objetivo de aprendizaje

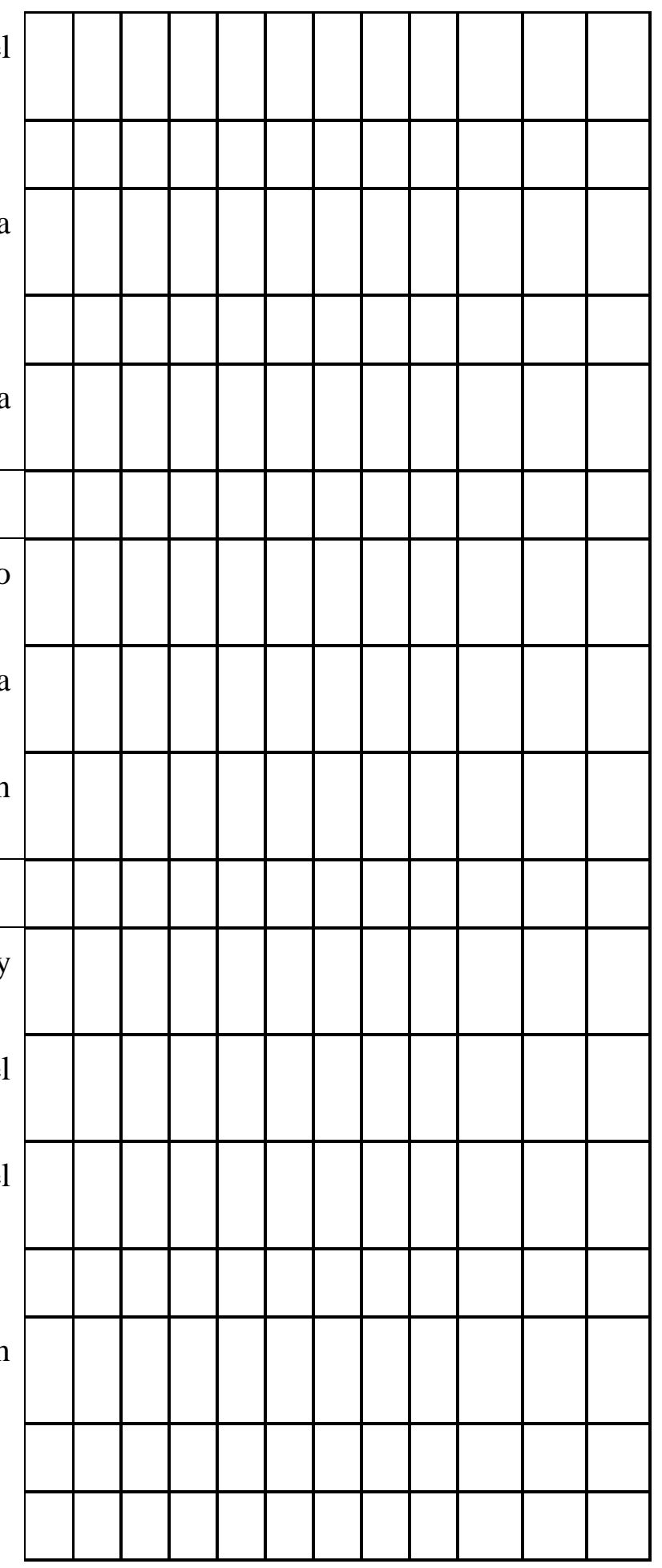

\section{ACTITUDES}

Expresó ideas de manera clara, organizada y coherente

Fue responsable en los acuerdos establecidos por e grupo

Demostró compromiso y respeto con el trabajo del grupo

Utilizó un lenguaje profesional

Interactuó de manera respetuosa y profesional con el grupo

\section{Subtotal}

\section{Calificación final}

\section{RELACIÓN DE ESTUDIANTES}

$\begin{array}{lll}1 .- & 2 .- & 3 .- \\ 4 .- & 5 .- & 6 .- \\ 7 .- & 8 .- & 9 .- \\ 10 .- & 11 .- & 12 .-\end{array}$

Escala: Excelente (2.5) Muy bien (2) Bien (1.5) Regular (1) Deficiente (0.5) No Aplica (NA):

Fuente: Facultad de Trabajo Social de la Universidad de Colima (2021) 


\section{Experiencias del ABP en la Universidad de Colima}

En este sentido parte Márquez et al (2011) en la facultad de Psicología y Medicina establecen que uno de los problemas de suma importancia es el proceso de evaluación, donde los alumnos aseguran estar menos satisfechos; los alumnos de Medicina fueron los menos satisfechos. Pese a las diferencias entre los de Psicología y Medicina, ambos indican su inconformidad por la subjetividad que cada tutor y ellos mismos imprimen en el proceso de evaluación; ello revela que, con base en su experiencia escolar previa, han venido visualizando la evaluación como sinónimo de calificación exclusiva del profesor y no necesariamente como un proceso formativo y de reflexión de su proceso de aprendizaje.

Más de $60 \%$ prefieran el $\mathrm{ABP}$ en su proceso de formación profesional sobre la enseñanza tradicional, deja claro que esta nueva forma de aprender, esta estrategia didáctica, es bien valorada por los estudiantes; por lo tanto, se tiene el compromiso de atender diversos aspectos: Destacar más los procesos de construcción del conocimiento, relacionando la actividad de la tutoría ABP con los aspectos metacognitivos del aprendizaje. Mayor claridad en los roles y funciones de los tutores en tutoría ABP. Dado su fundamento en el paradigma constructivista, el papel del tutor en la tutoría ABP es mediar el aprendizaje de su grupo. Claridad en la definición y evaluación de competencias, desentrándose de los contenidos y resaltando aspectos actitudinales, éticos, de habilidades comunicativas, de colaboración y trabajo en equipo, y Monitoreo constante en la actualización y capacitación docente para la formación de tutores y mejora de su desempeño.

En el mismo sentido Olmedo et al (2016) en el analisis de las Facultades de Medicina y Enfermería de la Universidad, mencionan que la experiencia que se ha tenido al trabajar con la metodología de ABP ha sido acertada, en el sentido de que los alumnos y docentes han desarrollado habilidades y competencias positivas, pero es cierto que se han tenido dificultades para su implementación y desarrollo, pero éstas se han ido superando con el paso de los años. Se tiene muy claro que el trabajo colegiado que se ha realizado año tras año y semestre tras semestre entre profesores de tutorías y de campos clínicos, ha permitido mejorar y adecuar no solo los contenidos teóricos de las tutorías académicas, sino también el grupo de actividades y habilidades prácticas que los alumnos realizan en sus campos clínicos y comunitarios; de tal forma que ahora están más cercanos a una realidad que le permitirá al estudiante poner en práctica sus habilidades y competencias acompañadas de un alto sentido de responsabilidad personal y profesional, humanística y ética como elementos básicos que requiere todo estudiante para tener éxito en el ABP y por ende en su vida profesional. 
Los requerimientos mínimos del ABP en la Universidad son: a) capacidad de resolver problemas o habilidades de razonamiento clínico, b) destrezas clínicas, c) habilidades para el autoaprendizaje, y d) habilidades de trabajo en equipo.

Si bien es cierto que el modelo favorece el desarrollo integral del estudiante, es importante considerar que el esquema mental sobre el proceso de enseñanza aprendizaje, las experiencias previas, así como las habilidades adquiridas en los niveles anteriores impactará en el éxito del estudiante en su proceso de formación profesional, sin olvidar el papel importante que juega el tutor para favorecer el aprendizaje en los estudiantes. Es importante saber hacia dónde debe dirigirse la educación superior y los métodos de enseñanza centrados en el estudiante que favorecen esas expectativas así como reconocer el gran reto que tiene el sistema educativo para que el egresado de educación superior tenga un alto grado de competitividad, siendo uno de los propósitos mejorar los métodos de enseñanza y aprendizaje en los niveles medio superior y superior para ofrecer profesionistas integrales de calidad a la sociedad.

\section{CONSIDERACIONES FINALES}

En difererentes aplicaciones del ABP se menciona que se invierte el camino convencional del proceso de aprendizaje; donde tradicionalmente primero se expone la información y posteriormente se busca la aplicación en la resolución de un problema, en el caso del ABP la primera opción es plantear o presentar el problema a los estudiantes, lo que ellos deben identificar sus necesidades de aprendizaje, posteriormente investigan y recopilan la información necesaria para finalmente regresar al problema y darle la solución.

Los resultados de la Universidad de Colima respecto a la implementación del ABP, se concluyen experiencias positivas como negativas, entre las positivas se resalta el trabajo colaborativo del profesorado, permite tener un acercamiento mejor entre profesor-alumno, por lo que la evaluación debe ser un método más de enseñanza, y también una manera real y directa de posibilitar el aprendizaje.

En el ABP, la evaluación se constituye en una herramienta de vital importancia por el cual se le otorga al estudiante la responsabilidad ser su propio evaluador en su proceso de aprendizaje y de formación. Esto se trata de visualizar la evaluación como un proceso en el que la responsabilidad es compartida por los estudiantes y profesores.

El valor de la evaluación en el ABP es el de contemplar la evaluación individualizada, cualitativa y formativa. El estudiante tiene la posibilidad de evaluarse a sí mismo, a sus compañeros, al tutor, al proceso de trabajo en equipo y a los resultados del proceso. Con un cambio sustancial un cambio sustancial en la práctica en relación con el método tradicional. 
Un punto importante al evaluar con rúbricas y que no se ha tomado en dichas evaluaciones, se debe tomar en cuenta en el contexto laboral-docente, es el tipo de alumno que se tiene, los ritmos, estilos y motivos diferentes de aprendizaje. Además, en el aula conviven estudiantes que llegan de diversas condiciones sociales, de hogares disfuncionales, algunos con paupérrimas condiciones de vida; otros de sociedades donde impera la delincuencia y todos los vicios sociales. El trabajo con los estudiantes requiere un manejo pedagógico y psicológico y mover estructuras mentales necesita constante preparación.

\section{LISTA DE REFERENCIAS}

Arends, R. (2007). Aprender a Enseñar. $7^{\circ}$ edición. México: McGraw-Hill.

Barrows H.S. (1986) A Taxonomy of problembased learning methods, Medical Education, 20: 481-486. https://doi.org/10.1111/j.1365-2923.1986.tb01386.x.

Boletines informativos de la Universidad de Colima. 2018. http://www.ucol.mx/boletines/index.php?idn=6351\&mes=1\&dia=31\&year=2008.

Cohen-Schotanus, J., Muijtjens, A. M. M., Schönrock-Adema, J., Geertsma, J. y Van der Vleuten, C. P. M. (2008). Effects of conventional and problem-based learning on clinical and general competencies and career development. Medical Education, 42, 256-265. DOI: $10.1111 /$ j.1365-2923.2007.02959.x

Egido, I., Aranda, R., Cerrillo, R., De la Herrán, A., De Miguel, S., Gómez, M., Hernández, R., Izuzquiza, D., Murillo, F. J., \& Pérez, M. (2006). Aprendizaje basado en problemas: Estrategia metodológica y organizativa del currículum para la calidad de la enseñanza en los estudios de Magisterio. Revista Interuniversitaria de Formación del Profesorado, 20(3), 137-149.

Gómez P., Del Pozo G..V.M., Muñoz R.E , Guijarro, E., Canós D.L, Santandre M.C. (2020.) Elementos clave para un modelo e aprendizaje basado en proyectos colaborativos online (ABPCL) en la Educación Superior, American Journal of Distance Education, 34: 3, 241-253, DOI: 10.1080 / 08923647.2020.1805225.

González H., Carbonero M.C., , Lara O.M Á., Villamor F. Villamor M.P. (2014). Aprendizaje Basado en Problemas y satisfacción de los estudiantes de Enfermería. Enfermería Global, 13(35), 97-104.

Jofré M, Cecilia, \& Contreras H, Fernando. (2013). Implementación de la Metodología ABP (Aprendizaje Basado en Problemas) en Estudiantes de Primer año de la Carrera de Educación Diferencial. Estudios $\quad$ pedagógicos $\quad$ (Valdivia), 39(1), 113. https://dx.doi.org/10.4067/S0718-07052013000100006. 
Koh, G. C., Khoo, H. E., Wong, M. L. y Koh, D. (2008). The effects of problem-based learning during medical school on physician competency: A systematic review. Canadian Medical Association Journal, 178(1), 34-41. doi: 10.1503 / cmaj.070565.

Mora V.A.I. (2004). La evaluación educativa: Concepto, períodos y modelos Revista Electrónica "Actualidades Investigativas en Educación", 4(2), 1-28.

Mosquera, J. C. \& Furió, C. (2008). El cambio didáctico en profesores universitarios de química a través de un programa de actividades basado en la enseñanza por investigación orientada. Didáctica de las Ciencias Experimentales y Sociales, 22, 115-154.

Olmedo-Buenrostro, B., Alvarado Banda, H., Delgado Enciso, I., Montero Cruz, S., Cadenas Freixas, J., Mora-Brambila, A., \& Hernández-Torres, E. (2016). Desempeño estudiantil con el aprendizaje basado en problemas: habilidades y dificultades. Revista Cubana De Medicina General Integral, 32(2), 1-12.

Prieto, A., Barbarroja, J., Reyes, El., Montserrat, J., Díaz, D., Villaroel, M. \& Álvarez-Mon, M. (2006). Un nuevo modelo de aprendizaje basado en problemas, el ABP 4x4, es eficaz para desarrollar competencias profesionales valiosas en asignaturas con más de 100 alumnos. Aula Abierta, 87, 171-194.

Rajab, A. M. (2007). The effects of problem-based learning on the self-efficacy and attitudes of beginning biology majors. ProQuest Information \& Learning. Dissertation Abstracts International Section A: Humanities and Social Sciences, 68(6-A), 2359-2359.

Rivera de Parada, A. (2006). Aprendizaje Basado en Problemas (ABP) Estrategia para dinamizar la cátedra universitaria. Crea Ciencia Revista Científica, 4, 29-35. https://doi.org/10.5377/creaciencia.v0i4.9161

Scaffa, M. E. y Wooster, D. M. (2004). Effects of problem-based learning on clinical reasoning in occupational therapy. American Journal of Occupational Therapy, 58, 333-336. https://doi.org/10.5014/ajot.58.3.333.

Sackett DL, Rosenberg MCW, Gray JA, Haynes RB, Scott RW. Evidence based medicine: what it is and what it isn't. BMJ. 1996;12:71-72.

Schmidt, H. G., Vermeulen, L. y van der Molen, H. T. (2006). Longterm effects of problem based learning: A comparison of competencies acquired by graduates of a problem -based and a conventional medical school. Medical Education, 40(6), 562-567. DOI: $10.1111 /$ j.13652929.2006.02483.x

Schmidth, H. et al (1987). "Comparing the effects of Problem-Based and Conventional Curricula in an International Sample”, en Journal of Medical Education, 62, 305-315. 
Şendağa, S. y Odabaşi, H. F. (2009). Effects of an online problem based learning course on content knowledge acquisition and critical thinking skills. Computers \& Education, 53(1), 132-141. http://dx.doi.org/10.1016/j.compedu.2009.01.008

Servín J.J. (2018). Modalidades didácticas para el desarrollo de competencias, aprendizaje basado en problemas (ABP). P, 84.

Sungur, S. y Tekkaya, C. (2010). Effects of problem-based learning and traditional instruction on self-regulated learning. Journal of Educational Research, 99(5), 307-320. https://doi.org/10.3200/JOER.99.5.307-320

Torp, L. Sage, S. (1999). El aprendizaje basado en problemas: desde el jardín de infantes hasta el final de la escuela secundaria. Buenos Aires: Amorrurtu. 